\title{
Radiation Impedance of Collapsed Capacitive Micromachined Ultrasonic Transducers
}

\author{
Alper Ozgurluk, Student Member, IEEE, Abdullah Atalar, Fellow, IEEE, \\ Hayrettin Köymen, Senior Member, IEEE, and Selim Olçum, Member, IEEE
}

\begin{abstract}
The radiation impedance of a capacitive micromachined ultrasonic transducer (CMUT) array is a critical parameter to achieve high performance. In this paper, we present a calculation of the radiation impedance of collapsed, clamped, circular CMUTs both analytically and using finite element method (FEM) simulations. First, we model the radiation impedance of a single collapsed CMUT cell analytically by expressing its velocity profile as a linear combination of special functions for which the generated pressures are known. For an array of collapsed CMUT cells, the mutual impedance between the cells is also taken into account. The radiation impedances for arrays of $7,19,37$, and 61 circular collapsed CMUT cells for different contact radii are calculated both analytically and by FEM simulations. The radiation resistance of an array reaches a plateau and maintains this level for a wide frequency range. The variation of radiation reactance with respect to frequency indicates an inductance-like behavior in the same frequency range. We find that the peak radiation resistance value is reached at higher $k d$ values in the collapsed case as compared with the uncollapsed case, where $k$ is the wavenumber and $d$ is the center-to-center distance between two neighboring CMUT cells.
\end{abstract}

\section{INTRODUCTION}

$\mathrm{C}$ APACITIVE micromachined ultrasonic transducers (CMUTs) [1], [2] have been of interest because of their wider bandwidth compared with piezoelectric transducers [3]. Medical imaging [4]-[6], high-intensity focused ultrasound (HIFU) treatment [7], [8], and intravascular ultrasound (IVUS) [9]-[11] are just a few of the application areas where they are currently being considered as a promising technology.

Analysis and design of CMUTs are performed by using finite element method (FEM) simulations [12]-[15] and electrical equivalent circuit models [16]-[19]. Because FEM simulations require a considerable amount of time, equivalent circuit modeling is preferable in the initial phase of a design. The radiation impedance terminating the acoustic port is a critical element of an equivalent circuit model. The radiation impedance determines how much acoustical power is transmitted to the surround-

Manuscript received November 20, 2011; accepted March 2, 2012. This work was supported by the Scientific and Technological Research Council of Turkey (TUBITAK) under project grant 110E216. A. Atalar acknowledges the support of the Turkish Academy of Sciences (TUBA).

A. Ozgurluk, A. Atalar, and H. Köymen are with and S. Olçum was with the Electrical and Electronics Engineering Department, Bilkent University, Ankara, Turkey (e-mail: olcum@mit.edu).

S. Olçum is now with the Department of Biological Engineering, Massachusetts Institute of Technology, Cambridge, MA.

DOI http://dx.doi.org/10.1109/TUFFC.2012.2321 ing medium given the motion of the CMUT plate [20], [21]. The real part of the radiation impedance represents the power radiated to the medium, whereas the imaginary part is related to the reactive energy stored in the near field. Some of the previous circuit modeling efforts assume a purely real and constant radiation impedance [22]. This assumption approximates the behavior of a CMUT cell in a sufficiently large array. However, for smaller CMUT arrays, a more accurate analysis of radiation impedance is needed for a better estimation of the array performance. In a recent paper, the radiation impedances of elements with different numbers of cells were calculated for conventional (uncollapsed) CMUTs [23].

Because of higher transmission sensitivity of CMUTs in collapsed state [13], [24]-[27], special attention has been drawn to operation modes where the CMUT plate is collapsed as in Fig. 1. In a recent paper, a model taking into account the non-linear effects in the collapsed state was presented [19]. This model approximates the behavior of collapsed and uncollapsed CMUTs when they are in a very large array, and the radiation impedance seen by each cell is assumed to be purely real. For analyzing a single CMUT or a relatively small collapsed CMUT array, an accurate calculation of the radiation impedance is needed.

In this work, we calculate the radiation impedance of an array of collapsed CMUTs using the approach suggested in [23]. As an initial step, the radiation impedance of a collapsed single cell CMUT is calculated both analytically and using FEM simulations. Then, the radiation impedance of an array of collapsed CMUT cells is calculated by including the mutual impedance between the cells.

In [19, Fig. 12], it is shown that at a given excitation voltage, there is an optimum gap height, $t_{\mathrm{gopt}}$, to generate the maximum transmitted power. This can be understood by considering a CMUT cell with a gap of $t_{\mathrm{g}}$. When $t_{\mathrm{g}}>$ $t_{\text {gopt }}$, the Coulomb force that this cell can generate is $F=$ $k_{1} / t_{\mathrm{g}}^{2}$ (as can be verified from the figure) where $k_{1}$ is a constant. The acoustic power delivered to medium is $P=$ $F^{2} / Z=k_{1}^{2} /\left(Z t_{\mathrm{g}}^{4}\right)$, where $Z$ is the radiation resistance. On the other hand, when $t_{\mathrm{g}}<t_{\mathrm{gopt}}$, it is in the velocity-limited region. In this region, there is an upper bound on the particle velocity for large-signal operation and the excitation voltage must be reduced as $t_{\mathrm{g}}$ is reduced. The maximum velocity is limited by $t_{\mathrm{g}}$, and we write $v=k_{2} t_{\mathrm{g}}$ (and hence, $F=k_{2} t_{\mathrm{g}} Z$ ), where $k_{2}$ is a constant and $P=Z v^{2}=$ $Z k_{2}^{2} t_{\mathrm{g}}^{2}$. The two power expressions must be equal to each other at $t_{\mathrm{g}}=t_{\mathrm{gopt}}$, producing $t_{\mathrm{gopt}}=\left(k_{1} / k_{2} Z\right)^{1 / 3}$ and $P_{\max }$ $=k_{1} k_{2} / t_{\mathrm{gopt}}=k_{1}^{2 / 3} k_{2}^{4 / 3} Z^{1 / 3}$. This means that at $t_{\mathrm{g}}=t_{\mathrm{gopt}}$, 


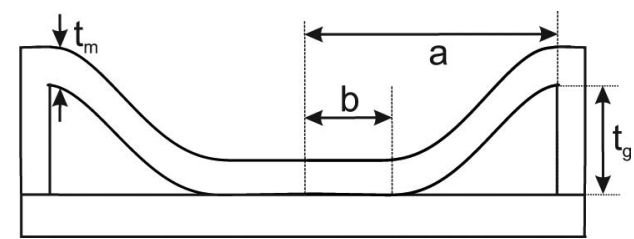

Fig. 1. Cross-sectional view of a collapsed CMUT cell with radius $a$, contact radius $b$, thickness $t_{m}$, and gap height $t_{\mathrm{g}}$.

$P_{\max } \propto Z^{1 / 3}$ and radiation resistance $Z$ must be made as high as possible to maximize the delivered power. We will show the conditions to reach a maximum in the radiation resistance.

\section{Single-Cell Radiation Impedance}

\section{A. Method Overview}

The radiation impedance of a circular CMUT cell is calculated by dividing the total power, $P$, on the surface of the CMUT cell to the square of the absolute value of the spatial $\mathrm{rms}$ velocity, $v_{\mathrm{R}}$, of the plate [20], [21]:

$$
Z=\frac{P}{\left|v_{\mathrm{R}}\right|^{2}}=\frac{\int_{S} p(r) v^{*}(r) \mathrm{d} S}{\left|v_{\mathrm{R}}\right|^{2}},
$$

where $p(r)$ and $v(r)$ are the pressure and the particle velocity on the surface, $S$, of the cell. We use the following definition for the spatial rms velocity, $v_{\mathrm{R}}$, as a complex number:

$$
v_{\mathrm{R}}=\sqrt{\frac{1}{S} \int_{S} \operatorname{Re}\{v(r)\}^{2} \mathrm{~d} S}+i \sqrt{\frac{1}{S} \int_{S} \operatorname{Im}\{v(r)\}^{2} \mathrm{~d} S} .
$$

The pressure generated on the surface of the plate by the velocity profile of the CMUT cell must be known to find the radiation impedance. However, it is not easy to find the pressure generated by an arbitrary velocity profile. To overcome this difficulty, we employ the same approach used in [23]. The actual velocity profile, $v(r)$, is expressed as a linear combination of the functions given by [28][30] for which the generated pressures on the surface are known. These functions are given as

$$
v_{n}(r)=v_{\mathrm{R}} \sqrt{2 n+1}\left(1-\left(\frac{r^{2}}{a^{2}}\right)\right)^{n} H(a-r),
$$

where $a$ is the radius of the radiator and $H$ is the unit step function. We use the function in (3) with $n=2,3,4,5$ and approximate the actual velocity profile, $v(r)$, obtained from the FEM simulations as

$$
v(r)=\alpha_{2} v_{2}(r)+\alpha_{3} v_{3}(r)+\alpha_{4} v_{4}(r)+\alpha_{5} v_{5}(r),
$$

where $\alpha_{n} \mathrm{~s}$ are real numbers. A constrained least-square algorithm is employed to obtain $\alpha_{n}$ values for the best fit at the frequency of interest. Because the velocity profile

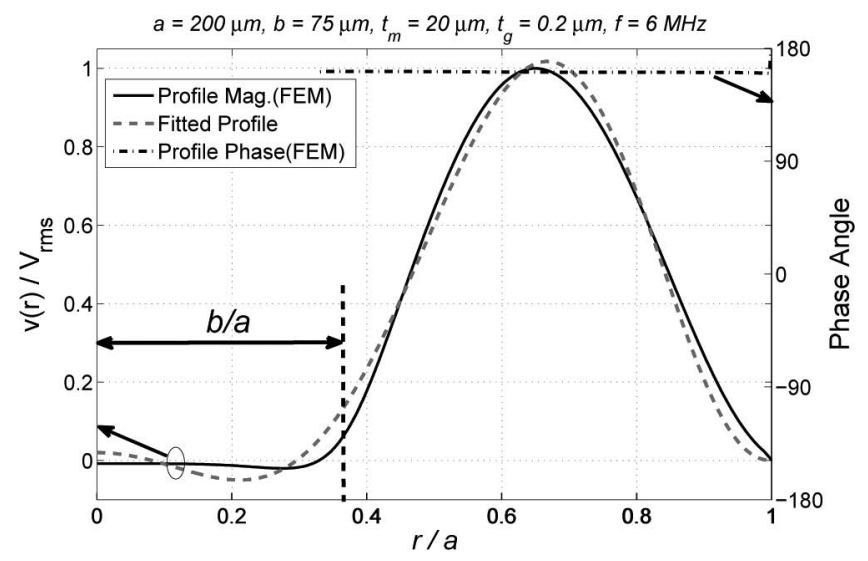

Fig. 2. Normalized velocity profile of a collapsed CMUT cell under a sinusoidal excitation taken through the cross section in Fig. 1 where $b$ is the contact radius and $a$ is the plate radius. Solid line represents the actual velocity profile and dashed line shows the fitted velocity profile for $\alpha_{2}=1.58, \alpha_{3}=15.63, \alpha_{4}=-32.51, \alpha_{5}=15.87$.

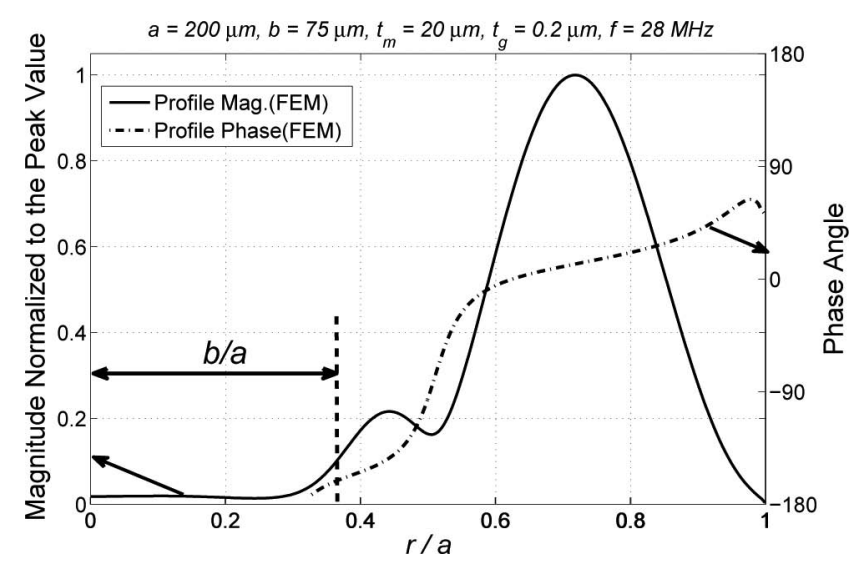

Fig. 3. Normalized velocity profile of a collapsed CMUT cell at a frequency greater than the antiresonance frequency.

depends on the frequency of excitation, different $\alpha_{n}$ values are found at different frequencies. As an example, the velocity profile of a collapsed CMUT cell under a sinusoidal excitation obtained by a prestressed harmonic FEM simulation and its approximation using (3) and (4) with optimized $\alpha_{n}$ values are plotted in Fig. 2 (see also Fig. 3). ${ }^{1}$

Having expressed the velocity profile in terms of the functions in (3), the total pressure on the surface of the CMUT cell can be found using the expressions in [28] which give the pressure generated by each of the velocity profiles in (3). The total pressure, $p(r)$, on the surface can be written as a linear combination of the pressures generated by each of the velocity profiles with the same weighting coefficients used in (4)

$$
p(r)=\alpha_{2} p_{2}(r)+\alpha_{3} p_{3}(r)+\alpha_{4} p_{4}(r)+\alpha_{5} p_{5}(r)
$$

${ }^{1}$ The fitting algorithm works satisfactorily if the operating frequency is not close to the antiresonance frequency of the plate. At or above the antiresonance frequency, the velocity profile does not have a constant phase, as seen in Fig. 3, and the fitting algorithm fails. 


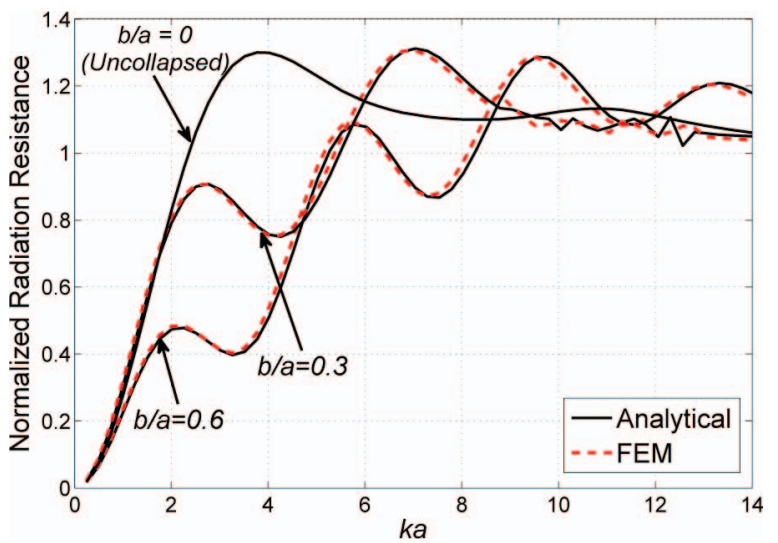

Fig. 4. Normalized radiation resistance as a function of $k a$ for a single cell CMUT $\left(a / t_{m}=5\right)$ in a collapsed state with different $b / a$ ratios.

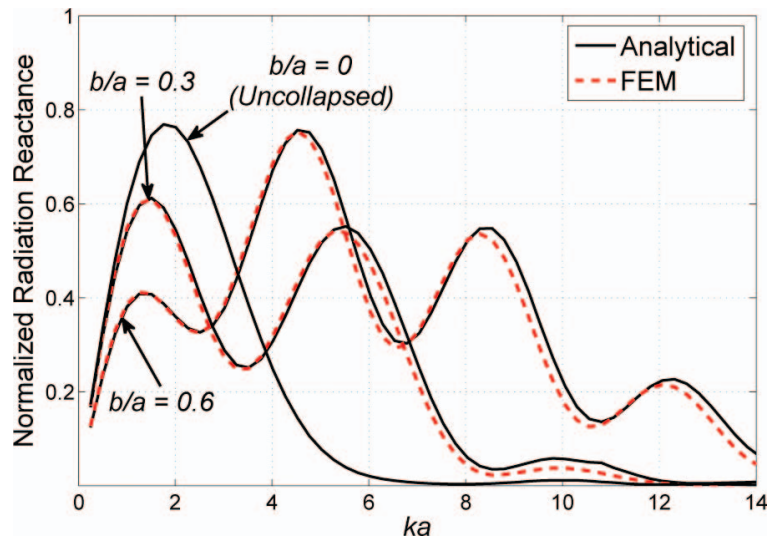

Fig. 5. Normalized radiation reactance as a function of $k a$ for a single cell CMUT $\left(a / t_{m}=5\right)$ in a collapsed state with different $b / a$ ratios.

where $p_{n}(r)$ is the pressure generated by $v_{n}(r)$.

Substituting (4) and (5) into (1) and using the approach suggested by [28], the radiation impedance can be found as

$$
Z=\frac{\sum_{n=2}^{5} \sum_{m=2}^{5} \alpha_{n} \alpha_{m} P_{n m}}{\left|v_{\mathrm{R}}\right|^{2}}
$$

and

$$
P_{n m}=S \rho_{0} c_{0}\left|v_{\mathrm{R}}\right|^{2} A\left\{1-B\left[F_{1 n m}(2 k a)+i F_{2 n m}(2 k a)\right]\right\},
$$

where $\rho_{0}$ is the density, $c_{0}$ is the speed of sound, and $k$ is the wavenumber of the immersion medium. Constants $(A$ and $B)$ and functions $\left(F_{1 n m}\right.$ and $\left.F_{2 n m}\right)$ are given in the Table I for $n, m=2,3,4,5$. In Table I, $J_{n}$ and $H_{n}$ are the $n$th order Bessel and Struve functions, respectively.

\section{B. Single-Cell Radiation Impedance}

Calculated radiation resistance and reactance curves normalized by $\rho_{0} c_{0} S$ for a relatively thick single collapsed CMUT cell are given in Figs. 4 and 5, respectively, along

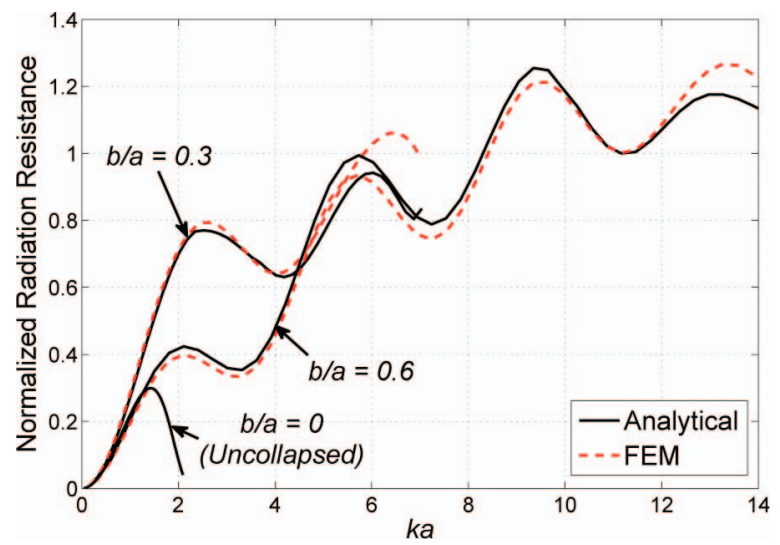

Fig. 6. Normalized radiation resistance as a function of $k a$ (up to the antiresonance frequency) for a single cell CMUT $\left(a / t_{m}=20\right)$ in a collapsed state with different $b / a$ ratios.

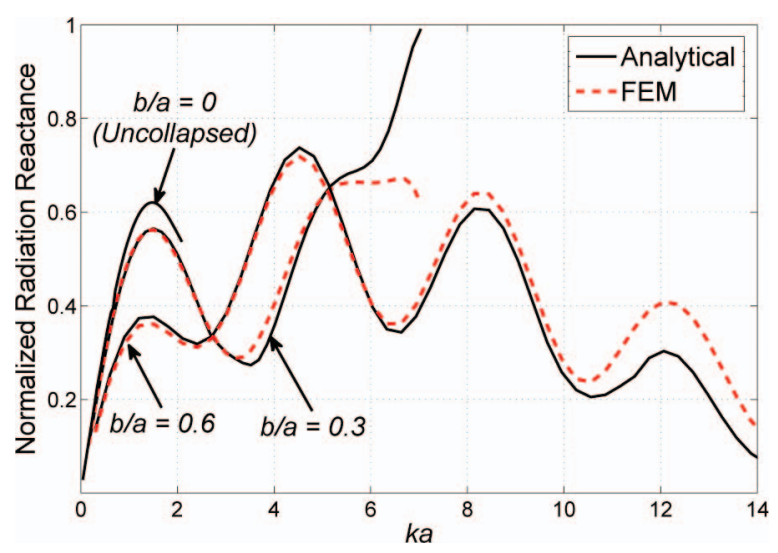

Fig. 7. Normalized radiation reactance as a function of $k a$ (up to the antiresonance frequency) for a single cell CMUT $\left(a / t_{m}=20\right)$ in a collapsed state with different $b / a$ ratios.

with the FEM simulation ${ }^{2}$ results. We note that such a thick plate gives accurate ${ }^{3}$ results for a wider range of $k a$ values. The radiation impedance of an uncollapsed CMUT plate is also depicted in the same figures. The material properties used in the simulations are given in Table II.

For $k a<5$, the radiation resistance in the collapsed state is less than that in the uncollapsed state. As the $b / a$ ratio increases, the radiation impedance reduces further. On the other hand, the radiation reactance in the collapsed state does not vanish even for $k a>6$. A similar behavior was also observed in the radiation impedance of a piston ring transducer [31].

Figs. 6 and 7 show how the radiation impedance of a collapsed CMUT cell behaves if a thinner plate is used. In

${ }^{2}$ FEM simulations were performed using Ansys (v13, Ansys Inc., Canonsburg, PA) constructing a model similar to that in [23]. TRANS126 elements of Ansys are also added to this model to enable the collapse of the plate as in [27].

${ }^{3}$ The calculation of the radiation impedance using analytical and FEM simulation methods may be prone to the same source of error. This is because both methods use the same velocity profiles obtained from FEM simulations because there is no analytical expression available for the velocity profile of a collapsed CMUT plate. 


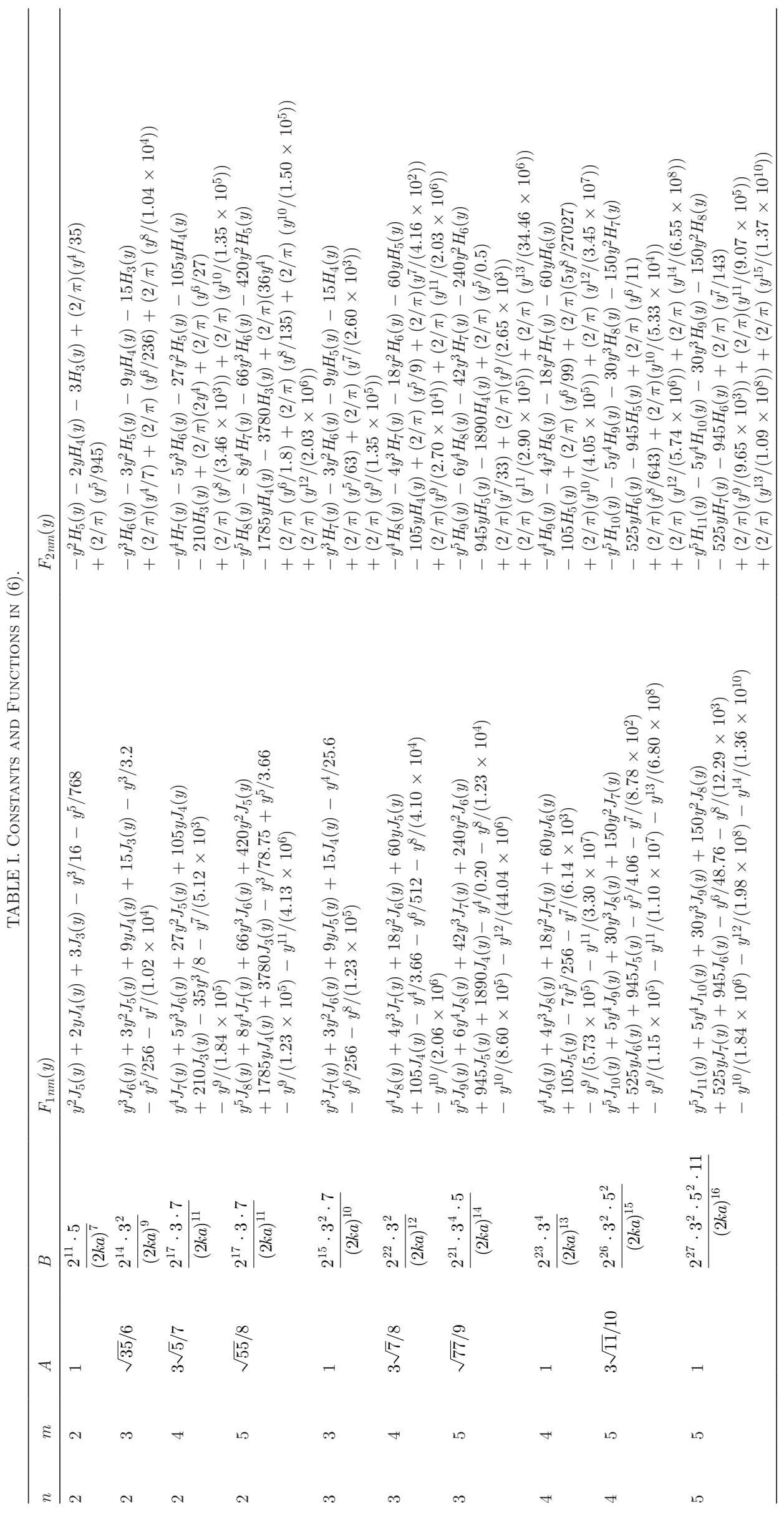




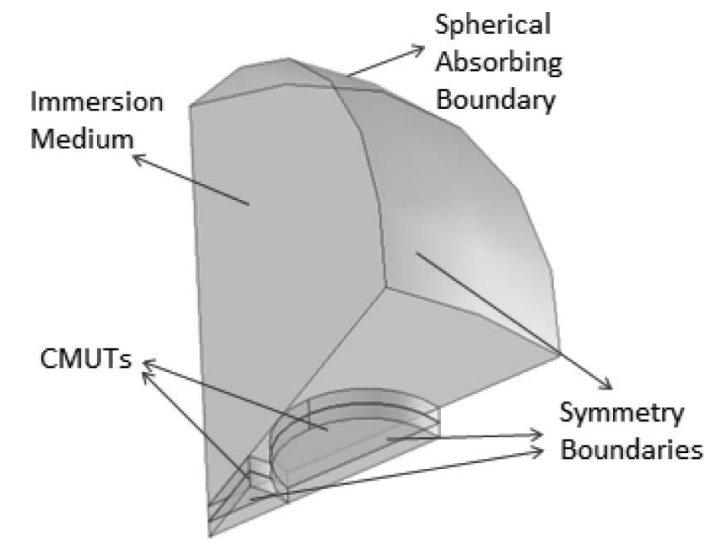

Fig. 8. 3-D symmetric finite element model of an array with 7 cells.

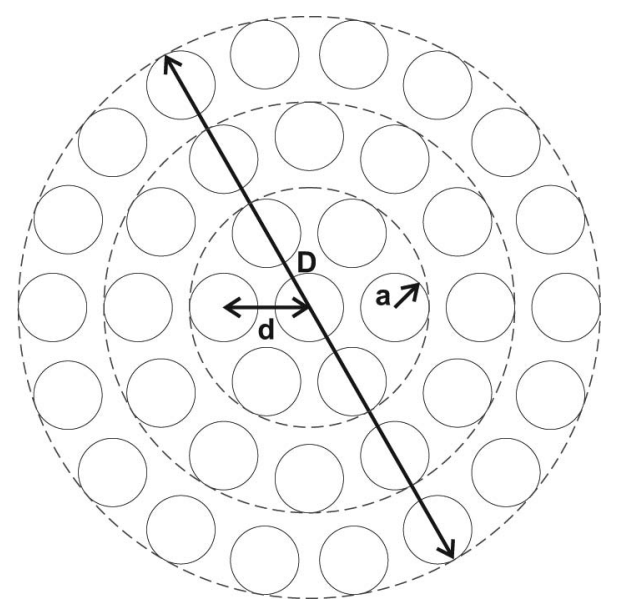

Fig. 9. Geometry of an array considered in this study with 37 cells.

the figures, the radiation impedance is plotted up to the antiresonance frequency. Because this plate is thinner, its antiresonance frequency is lower compared with the previous case, but increases as the contact radius, $b$, increases. The radiation impedance of a thinner plate is the same as that of the thicker plate at lower frequencies and deviates from it as the frequency approaches the antiresonance frequency.

\section{Array Radiation Impedance}

\section{A. FEM Simulations}

3-D FEM simulations for an array of collapsed CMUT cells are performed in Comsol Multiphysics (v4.0a, Comsol Inc., Providence, RI, http://www.comsol.com) using one-twelfth of the overall 7-cell structure (Fig. 8) with appropriate symmetry boundary conditions. Solid mechanics and pressure acoustics modules are utilized and tied together through the acoustic pressure and acceleration on the top surface of the plate. A spherical absorbing boundary is placed $2 \lambda_{0}$ away from the plate and the maximum mesh size is selected to be $\lambda_{0} / 10$, where $\lambda_{0}$ is the wave-
TABle II. Material Properties Used in the Simulations.

\begin{tabular}{lcc}
\hline Parameter & $\begin{array}{c}\text { CMUT } \\
\text { plate }\end{array}$ & $\begin{array}{c}\text { Immersion } \\
\text { medium }\end{array}$ \\
\hline $\begin{array}{l}\text { Density }\left(\mathrm{kg} / \mathrm{m}^{3}\right) \\
\text { Poisson's ratio }\end{array}$ & 3270 & $\rho_{0}=1000$ \\
Speed of sound $(\mathrm{m} / \mathrm{s})$ & 0.263 & $c_{0}=1500$ \\
Young's modulus $(\mathrm{GPa})$ & 320 & \\
\hline
\end{tabular}

length in the immersion medium. A contact definition is introduced between the top and bottom plates of CMUTs by selecting the bottom boundary of the top plate and top boundary of the bottom plate as source and destination boundaries, respectively. ${ }^{4}$

\section{B. Method Overview}

We consider arrays of $7,19,37$, and 61 cells placed as shown in Fig. 9. Each cell experiences an acoustic loading from the neighboring cells; hence, the mutual impedances between the cells must be taken into account. The mutual impedance, $Z_{i j}$, between the two CMUT cells is defined as

$$
Z_{i j}=\frac{P_{i j}}{v_{\mathrm{R} i} v_{\mathrm{R} j}^{*}}=\frac{\int_{S_{j}} p_{i j}(r) v_{i j}^{*}(r) \mathrm{d} S}{v_{\mathrm{R} i} v_{\mathrm{R} j}^{*}},
$$

where $P_{i j}$ is the power generated on surface of the $j$ th cell resulting from the pressure, $p_{i j}(r)$, and particle velocity, $v_{i j}(r)$, generated by the $i$ th cell. $v_{\mathrm{R} i}$ and $v_{\mathrm{R} j}$ are the spatial rms velocities of the $i$ th and $j$ th cells [21], respectively. $Z_{i j}$ can be written as

$$
Z_{i j}=\sum_{n=2 m=2}^{5} \sum_{n}^{5} \alpha_{n} \alpha_{m} Z_{i j}^{n m},
$$

where $Z_{i j}^{n m}$ is the mutual impedance between the $i$ th and $j$ th transducers having the velocity profiles $v_{n}(r)$ and $v_{m}(r)$ in (3). $Z_{i j}^{n m}$ is given by Porter [29] as an infinite summation.

Because of the symmetry, all CMUT cells placed in the same tier of an array have the same radiation impedance. The radiation impedance of cells located in different tiers will be different. To reduce the complexity, we prefer to define a representative radiation impedance for the whole array as

$$
Z_{\mathrm{r}}=\frac{\sum_{i=1}^{N} \int_{S_{i}} p_{i}(r) v_{i}^{*}(r) \mathrm{d} S}{\sum_{i=1}^{N}\left|v_{\mathrm{R} i}\right|^{2}},
$$

where $p_{i}(r)$ and $v_{i}(r)$ are the pressure and the particle velocity on the surface, $S_{i}$, of the $i$ th cell. $N$ is the total

\footnotetext{
${ }^{4}$ Comsol uses a penalty factor (similar to contact stiffness factor in Ansys) which is increased by default at each iteration to deal with the contact problems. However, this approach led to convergence problems. We chose the penalty factor to be a constant to overcome the problems.
} 


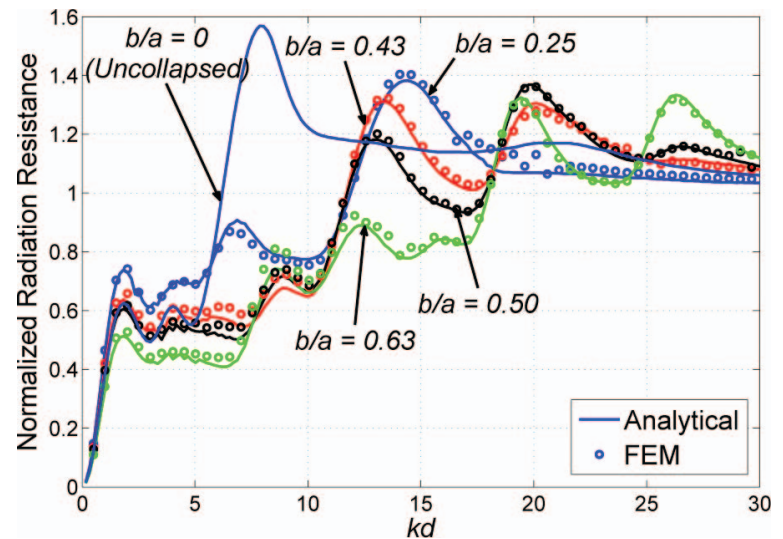

Fig. 10. Normalized radiation resistance for a 7-cell closely packed collapsed CMUT array with $a / t_{m}=5$ for different $b / a$. For comparison, the radiation resistance in an uncollapsed regime is also included.

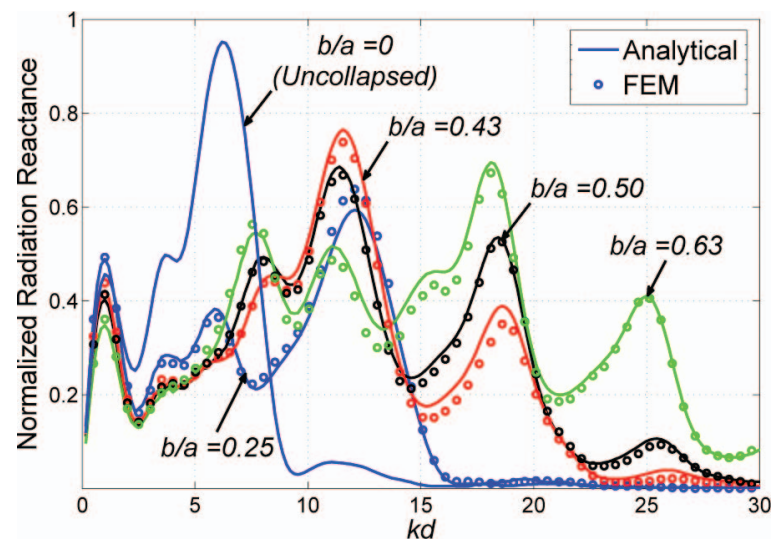

Fig. 11. Normalized radiation reactance for a 7-cell closely packed collapsed CMUT array with $a / t_{m}=5$ for different $b / a$. For comparison, radiation impedance in an uncollapsed regime is also included.

number of cells in the array. This impedance corresponds to the average radiation impedance of a single CMUT cell.

\section{Representative Radiation Impedance of an Array}

We present two sets of results for the representative radiation impedance of an array of collapsed CMUTs. In Figs. 10 and 11, the contact radius, $b$, is varied for a closely packed $(a / d=0.50)$ array of $N=7$. The normalization constant in all figures is $\rho_{0} c_{0} S$ where $S$ is the area of a single cell. The radiation impedance of the uncollapsed CMUT array [23] is also included in the figures for comparison. We find that a smaller peak radiation resistance value is reached at higher $k d$ values in the collapsed state as compared with the uncollapsed state.

For $k d<5$, the radiation resistance values for the uncollapsed and collapsed states are nearly the same. However, for $5<k d<10$, the radiation resistance in collapsed state becomes significantly less than that in the uncollapsed case. In addition, with increasing contact radius, the radiation resistance decreases for $k d<20$ and reaches a value of 0.5 for $1<k d<8$ when the contact radius be-

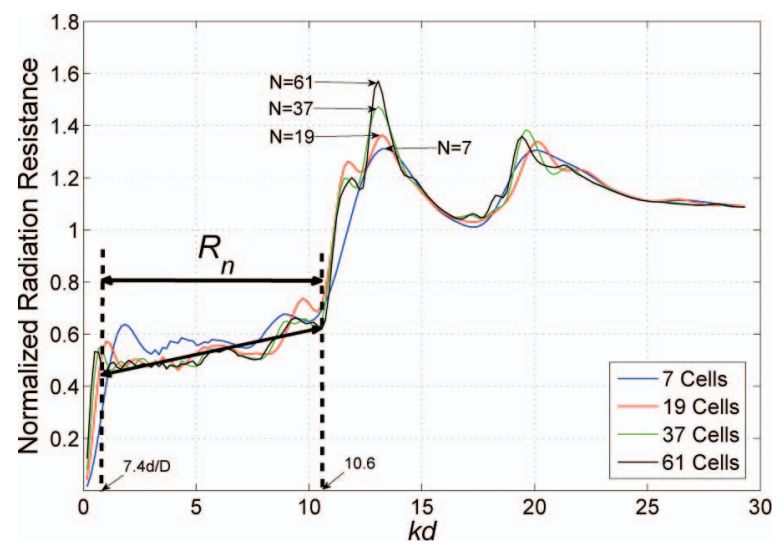

Fig. 12. Normalized radiation resistance for an array of collapsed CMUT cells with $N=7,19,37$, and 61 cells for $b / a=0.37, a / d=0.50$, and $a /$ $t_{m}=5$.

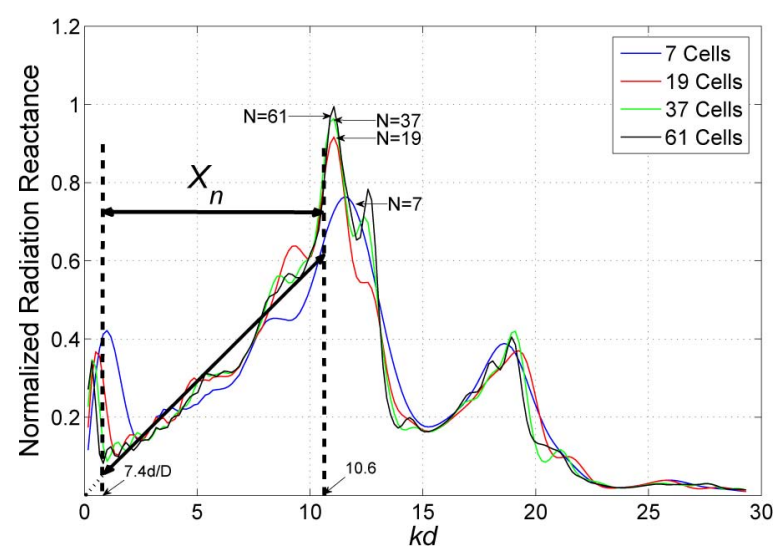

Fig. 13. Normalized radiation reactance for an array of collapsed CMUT cells with $N=7,19,37$, and 61 cells for $b / a=0.37, a / d=0.50$, and $a /$ $t_{m}=5$.

comes half of the radius of the CMUT cell. We also note that as $b / a$ is increased, the $k d$ range in which the radiation reactance is nonzero expands.

In the second set of results plotted in Figs. 12 and 13, the contact radius is kept constant at a nominal value $(b / a$ $=0.37)$. The representative radiation impedance of a collapsed array $(a / d=0.50)$ is investigated as the number of tiers in the array is changed from two to five, corresponding to $7,19,37$, and 61 cells. Employing a least-square fitting algorithm, the normalized radiation resistance of the array can be approximated by $R_{n} \approx 0.02 k d+0.42$ for $7.4 d / D<k d<10.6$. Here, $D$ represents the total diameter of the array as depicted in Fig. 9. Note that this range covers many practical CMUTs and for arrays with more cells, the radiation resistance obeys this linear relation for lower $k d$ values. The peak value of the radiation resistance is reached at about $k d=14.5$ and this peak value increases as the number of cells in the array increases. Similarly, the normalized radiation reactance can be approximated by $X_{n} \approx 0.06 k d$ for $7.4 d / D<k d<10.6$. Such a reactance can be represented by an inductance of $L=0.19 \rho_{0} d a^{2}$ in the electrical equivalent circuit. In practice, $a / d=0.50$ is 


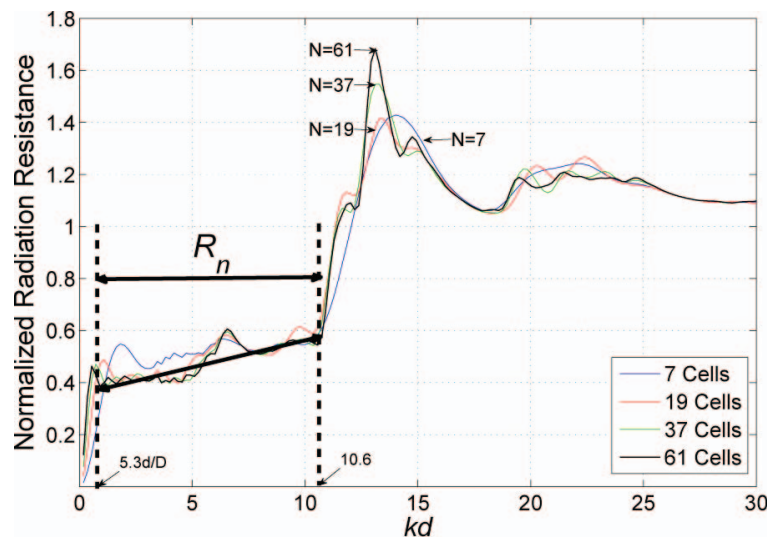

Fig. 14. Normalized radiation resistance for an array of collapsed CMUT cells with $N=7,19,37$, and 61 cells for $b / a=0.37, a / d=0.46$, and $a /$ $t_{m}=5$.

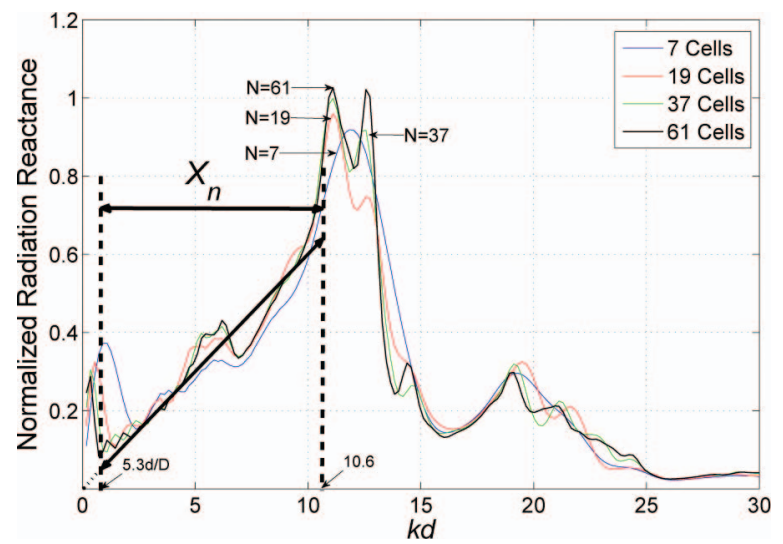

Fig. 15. Normalized radiation reactance for an array of collapsed CMUT cells with $N=7,19,37$, and 61 cells for $b / a=0.37, a / d=0.46$, and $a /$ $t_{m}=5$.

not possible, because there must be a nonzero kerf size between two neighboring CMUT cells. In [27], a kerf of $5 \mu \mathrm{m}$ was used for an array of CMUT cells with a plate radius of $30 \mu \mathrm{m}$, resulting in $a / d=0.46$. In Figs. 14 and 15 , the radiation impedance results for $a / d=0.46$ can be seen. ${ }^{5}$ In this case, we can write the approximate relations using the same fitting algorithm as $R_{n} \approx 0.02 k d+0.36$ and $X_{n}$ $\approx 0.06 k d$ for $5.3 d / D<k d<10.6$.

\section{CONCLusions}

In this work, the radiation impedance of an array of collapsed CMUTs was determined. First, the radiation impedance of a single collapsed CMUT cell for different contact radii was calculated. It was found that for a single cell with $a<\lambda_{0} / 2$, the radiation resistance and reactance decrease as the contact radius increases. For $a>2 \lambda_{0}$, the

\footnotetext{
${ }^{5}$ For fixed $d$, a smaller $a$ gives a higher normalized peak radiation resistance. However, the actual radiation resistance found after multiplication with the normalization constant $\left(\rho_{0} c_{0} \pi a^{2}\right)$ is smaller.
}

radiation impedance for a collapsed cell approaches that of an uncollapsed cell, if the collapse radius is not too large.

We also calculated the radiation impedance of an array of CMUT cells by taking into account the mutual impedance between the neighboring cells. Arrays of 7, 19, 37 , and 61 cells placed in a hexagonal pattern to form a circular transducer were considered. We found simple approximate relations for the radiation impedance valid when $0.2 \lambda_{0}<d<1.7 \lambda_{0}$. In this range, the normalized radiation resistance is roughly 0.5 and it is not highly dependent on the degree of collapse. In the same range, the radiation reactance can be approximated by an inductor.

\section{REFERENCES}

[1] H. T. Soh, I. Ladabaum, A. Atalar, C. F. Quate, and B. T. KhuriYakub, "Silicon micromachined ultrasonic immersion transducers," Appl. Phys. Lett., vol. 69, no. 24, pp. 3674-3676, 1996.

[2] I. Ladabaum, X. Jin, H. T. Soh, A. Atalar, and B. T. Khuri-Yakub, "Surface micromachined capacitive ultrasonic transducers," IEEE Trans. Ultrason. Ferroelectr. Freq. Control, vol. 45, no. 3, pp. 678690, 1998

[3] R. O. Guldiken, J. Zahorian, F. Y. Yamaner, and F. L. Degertekin, "Dual-electrode CMUT with non-uniform membranes for high electromechanical coupling coefficient and high bandwidth operation," IEEE Trans. Ultrason. Ferroelectr. Freq. Control, vol. 56, no. 6, pp. 1270-1276, 2009.

[4] J. A. Johnson, Ö. Oralkan, U. Demirci, S. Ergun, M. Karaman, and B. T. Khuri-Yakub, "Medical imaging using capacitive micromachined ultrasonic transducer arrays," Ultrasonics, vol. 40, no. 1-8, pp. 471-476, 2002.

[5] Ö. Oralkan, A. S. Ergun, J. A. Johnson, M. Karaman, U. Demirci, K. Kaviani, T. H. Lee, and B. T. Khuri-Yakub, "Capacitive micromachined ultrasonic transducers: Next-generation arrays for acoustic imaging?" IEEE Trans. Ultrason. Ferroelectr. Freq. Control, vol 49, no. 11, pp. 1596-1610, 2002.

[6] I. O. Wygant, X. Zhuang, D. T. Yeh, Ö. Oralkan, A. S. Ergun, M. Karaman, and B. T. Khuri-Yakub, "Integration of 2D CMUT arrays with front-end electronics for volumetric ultrasound imaging," IEEE Trans. Ultrason. Ferroelectr. Freq. Control, vol. 55, no. 2, pp. 327-342, 2008.

[7] S. H. Wong, R. D. Watkins, M. Kupnik, K. B. Pauly, and B. T. Khuri-Yakub, "Feasibility of MR-temperature mapping of ultrasonic heating from a CMUT," IEEE Trans. Ultrason. Ferroelectr. Freq. Control, vol. 55, no. 4, pp. 811-818, 2008.

[8] S. H. Wong, M. Kupnik, R. D. Watkins, K. Butts-Pauly, and B. T. Khuri-Yakub, "Capacitive micromachined ultrasonic transducers for therapeutic ultrasound applications," IEEE Trans. Biomed. Eng., vol. 57, no. 1, pp. 114-123, 2010.

[9] F. L. Degertekin, R. O. Guldiken, and M. Karaman, "Annular-ring CMUT arrays for forward-looking IVUS: Transducer characterization and imaging," IEEE Trans. Ultrason. Ferroelectr. Freq. Control, vol. 53, no. 2, pp. 474-482, 2006.

[10] X. Zhuang, D. S. Lin, Ö. Oralkan, and B. T. Khuri-Yakub, "Fabrication of flexible transducer arrays with through-wafer electrical interconnects based on trench refilling with PDMS," J. Microelectromech. Syst., vol. 17, no. 2, pp. 446-452, 2008.

[11] A. Nikoozadeh, Ö. Oralkan, M. Gencel, J. W. Choe, D. N. Stephens, A. de la Rama, P. Chen, K. Themenius, A. Dentinger, D. Wildes, K. Shivkumar, A. Mahajan, M. O'Donnell, D. Sahn, and P. T. Khuri-Yakub, "Forward-looking volumetric intercardiac imaging using fully integrated CMUT ring array," in Proc. IEEE Ultrasonics Symp., 2009, pp. 511-514.

[12] A. Bozkurt, I. Ladabaum, A. Atalar, and B. T. Khuri-Yakub, "Theory and analysis of electrode size optimization for capacitive microfabricated ultrasonic transducers," IEEE Trans. Ultrason. Ferroelectr. Freq. Control, vol. 46, no. 6, pp. 1364-1374, 1999.

[13] B. Bayram, E. Haeggstrom, G. G. Yaralioglu, and B. T. KhuriYakub, "A new regime for operating capacitive micromachined ul- 
trasonic transducers," IEEE Trans. Ultrason. Ferroelectr. Freq. Control, vol. 50, no. 9, pp. 1184-1190, 2003.

[14] G. G. Yaralioglu, A. S. Ergun, and B. T. Khuri-Yakub, "Finite-element analysis of capacitive micromachined ultrasonic transducers," IEEE Trans. Ultrason. Ferroelectr. Freq. Control, vol. 52, no. 12, pp. $2185-2198,2005$.

[15] B. Bayram, M. Kupnik, G. G. Yaralioglu, A. S. Ergun, D. Lin, S. H. Wong, and B. T. Khuri-Yakub, "Finite element modeling and experimental characterization of crosstalk in 1-D CMUT arrays," IEEE Trans. Ultrason. Ferroelectr. Freq. Control, vol. 54, no. 2, pp. 418-430, 2007.

[16] A. Lohfink and P.-C. Eccardt, "Linear and nonlinear equivalent circuit modeling of CMUTs," IEEE Trans. Ultrason. Ferroelectr. Freq. Control, vol. 52, no. 12, pp. 2163-2172, 2005.

[17] H. Köymen, M. N. Senlik, A. Atalar, and S. Olcum, "Parametric linear modeling of circular CMUT membranes in vacuum," IEEE Trans. Ultrason. Ferroelectr. Freq. Control, vol. 54, no. 6, pp. 1229 1239, 2007.

[18] H. K. Oguz, S. Olcum, M. N. Senlik, V. Tas, A. Atalar, and H. Köymen, "Nonlinear modeling of an immersed transmitting capacitive micromachined ultrasonic transducer for harmonic balance analysis," IEEE Trans. Ultrason. Ferroelectr. Freq. Control, vol. 57, no. 2, pp. $438-447,2010$.

[19] S. Olcum, F. Y. Yamaner, A. Bozkurt, H. Köymen, and A. Atalar, "An equivalent circuit model for transmitting capacitive micromachined ultrasonic transducers in collapse mode," IEEE Trans. Ultrason. Ferroelectr. Freq. Control, vol. 58, no. 7, pp. 1468-1477, 2011.

[20] L. L. Foldy, "Theory of passive linear electroacoustic transducers with fixed velocity distribution," J. Acoust. Soc. Am., vol. 21, no. 1, pp. 595-604, 1949.

[21] C. H. Sherman, "Analysis of acoustic interactions in transducer arrays," IEEE Trans. Sonics Ultrason., vol. 13, no. 1, pp. 9-15, 1966.

[22] S. Olcum, M. N. Senlik, and A. Atalar, "Optimization of the gainbandwidth product of capacitive micromachined ultrasonic transducers," IEEE Trans. Ultrason. Ferroelectr. Freq. Control, vol. 52, no. 12, pp. 2211-2219, 2005.

[23] M. N. Senlik, S. Olcum, H. Köymen, and A. Atalar, "Radiation impedance of an array of circular capacitive micromachined ultrasonic transducers," IEEE Trans. Ultrason. Ferroelectr. Freq. Control, vol. 57, no. 4, pp. 969-976, 2010.

[24] B. Bayram, Ö. Oralkan, A. S. Ergun, E. Hæggström, G. G. Yaralioglu, and B. T. Khuri-Yakub, "Capacitive micromachined ultrasonic transducer design for high power transmission," IEEE Trans. Ultrason. Ferroelectr. Freq. Control, vol. 52, no. 2, pp. 326-339, 2005.

$25]$ Ö. Oralkan, B. Bayram, G. G. Yaralioglu, A. S. Ergun, M. Kupnik, D. T. Yeh, I. O. Wygant, and B. T. Khuri-Yakub, "Experimental characterization of collapse-mode CMUT operation," IEEE Trans. Ultrason. Ferroelectr. Freq. Control, vol. 53, no. 8, pp. 1513-1523, 2006.

[26] Y. Huang, E. Haeggstrom, B. Bayram, X. Zhuang, A. S. Ergun, C.-H. Cheng, and B. T. Khuri-Yakub, "Comparison of conventional and collapsed region operation of capacitive micromachined ultrasonic transducers," IEEE Trans. Ultrason. Ferroelectr. Freq. Control, vol. 53, no. 10, pp. 1918-1933, 2006.

[27] S. Olcum, F. Y. Yamaner, A. Bozkurt, H. Köymen, and A. Atalar, "Deep-collapse operation of capacitive micromachined ultrasonic transducers," IEEE Trans. Ultrason. Ferroelectr. Freq. Control, vol. 58, no. 11 , pp. $2475-2483,2011$.

[28] M. Greenspan, "Piston radiator: Some extensions of the theory," $J$. Acoust. Soc. Am., vol. 65, no. 3, pp. 608-621, 1979.

[29] D. T. Porter, "Self-and mutual-radiation impedance and beam patterns for flexural disks in a rigid plane," J. Acoust. Soc. Am., vol. 36, no. 6, pp. 1154-1161, 1964.

[30] I. O. Wygant, M. Kupnik, and B. T. Khuri-Yakub, "Analytically calculating membrane displacement and the equivalent circuit model of a circular CMUT cell," in Proc. IEEE Ultrasonics Symp., 2008, pp. $2111-2114$.

[31] P. R. Stepanishen, "Impulse response and radiation impedance of an annular piston," J. Acoust. Soc. Am., vol. 56, no. 2, pp. 305-312, 1974 .

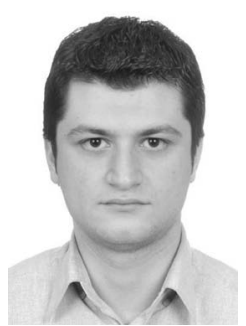

Alper Ozgurluk was born in Kirikkale, Turkey, in 1990. He is currently a senior undergraduate student in the Electrical and Electronics Engineering Department, Bilkent University, Ankara, Turkey.

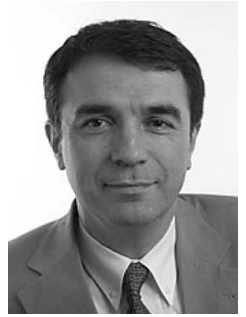

Abdullah Atalar received the B.S. degree from Middle East Technical University, Ankara, Turkey, in 1974 and M.S. and Ph.D. degrees from Stanford University, Stanford, CA, in 1976 and 1978, respectively, all in electrical engineering. He worked in Hewlett-Packard Labs, Palo Alto, in 1979. From 1980 to 1986, he was on the faculty of the Middle East Technical University as an Assistant Professor. In 1986, he joined Bilkent University as the chairman of the Electrical and Electronics Engineering Department and served in the founding of the department, where he is currently a Professor. In 1995 , he was a Visiting Professor at Stanford University. From 1996 to 2010, he was the Provost of Bilkent University. He is presently the Rector of the same university. His current research interests include micromachined devices and microwave electronics.

Prof. Atalar was awarded the Science Award of the Scientific and Technological Research Council of Turkey (TUBITAK) in 1994. He is a Fellow of IEEE and a member of the Turkish Academy of Sciences.

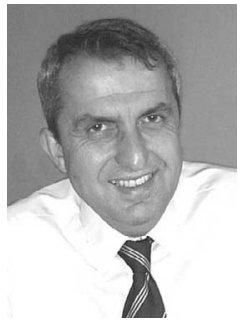

Hayrettin Köymen received the B.Sc. and M.Sc. degrees from Middle East Technical University (METU), Ankara, Turkey, in 1973 and 1976. respectively, and the Ph.D. degree from Birmingham University, UK, in 1979, all in electrical engineering. He worked as a faculty member in the Marine Sciences Department (Mersin) and Electrical Engineering Department (Ankara) of METU from 1979 to 1990, and at Bilkent University since 1990, where he is a professor. His research activities have included underwater acoustic and ultrasonic transducer design, acoustic microscopy, ultrasonic NDT, biomedical instrumentation, mobile communications, and spectrum management.

Prof. Köymen is a fellow of IET (formerly IEE).

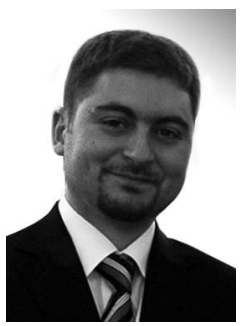

Selim Olçum was born in Chicago, IL, in 1981. He received his B.S., M.S., and Ph.D. degrees in electrical engineering in 2003, 2005, and 2010, respectively, all from Bilkent University, Ankara, Turkey. He worked as a guest researcher at the National Institute of Standards and Technology, Semiconductor Electronics Division, Gaithersburg, MD, during the summers of 2002 and 2003. He was a visiting scholar in the Micromachined Sensors and Transducers Laboratory of the Georgia Institute of Technology, Atlanta, GA, in 2006. He was an instructor in the Electrical and Electronics Engineering Department at Bilkent University for six months in 2011. He is currently a postdoctoral associate in Department of Biological Engineering and Koch Institute for Integrative Cancer Research at Massachusetts Institute of Technology, Cambridge, MA. His dissertation work was focused on developing high-performance micromachined ultrasonic transducers. His current research focus at MIT is to develop real-time techniques for bimolecular detection using micro- and nano-electromechanical devices.

Dr. Selim Olçum was a fellow of Aselsan during his Ph.D. study. 